\title{
Renal transplantation in ANCA-associated vasculitis
}

\author{
Mårten Segelmark
}

\section{Linköping University Post Print}

\section{Tweet}

N.B.: When citing this work, cite the original article.

Original Publication:

Mårten Segelmark, Renal transplantation in ANCA-associated vasculitis, 2013, La Médicale, (42), 4, 568-571.

http://dx.doi.org/10.1016/j.lpm.2013.01.023

Copyright: Elsevier Masson

http://www.elsevier-masson.fr/

Postprint available at: Linköping University Electronic Press

http://urn.kb.se/resolve?urn=urn:nbn:se:liu:diva-105257 


\title{
Renal transplantation in ANCA associated vasculitis
}

\author{
Mårten Segelmark, MD, PhD
}

Department of Medical and Health Sciences, Linköping University and Department of Nephrology, University Hospital UHL, County Council of Östergötland, Sweden.

marten.segelmark@liu.se

Renal transplantation is the treatment of choice for end-stage renal disease. It provides better results with respect to patient survival and morbidity as well as it offers superior possibilities for rehabilitation as compared to various dialysis modalities(1). Since renal engagement, often healing with scarring, is common in anti-neutrophil cytoplasm antibody (ANCA) associated vasculitis (AAV) (2), renal transplantation is a therapeutic option that frequently needs to be considered in AAV patients.

There are several publications clearly indicating that AAV patients do fairly well after renal transplantation and that AAV should definitely not be considered as a contra-indication for transplantation(3-12). However, there are several issues regarding the treatment of AAV before and after transplantation that remain unresolved which will be addressed in this brief review. These issues include the epidemiology of AAV and transplantation, timing of transplantation, risk of relapse, role of ANCA measurements and treatment of relapses after transplantation.

\section{Scope of the problem}

There are limited data on the number AAV patients having received a renal transplant and as well as numbers on waiting lists. Understanding of the epidemiology of AAV in end-stage renal disease is hampered by unresolved matters regarding nomenclature and classification of vasculitis(13). Before the age of prevalent ANCA testing many patients with AAV went into renal failure without a distinct diagnosis. Weidemann et al reported in 1993 on ANCA among 1277 dialysis patients, about $7 \%$ were positive and a distinct AAV diagnosis could retrospectively be made in a substantial portion of them(14). Many European renal registries use the EDTA codes where patients with renal limited AAV 
(RLV) could be classified as either "crescentic glomerulonephritis" (EDTA code 17) or "glomerulonephritis not described above" (code 19) depending on the severity of the glomerular lesions, and if no biopsy is performed as "unknown" (code 00). AAV patients with extra-renal symptoms could be listed as either "Wegener's Granulomatosis" (code 74), "renal vascular disease due to polyarthritis" (code 73) or "other multisystem disease" (code 89). No meaningful epidemiological data on AAV and end-stage renal disease can be extracted from of registries using EDTA codes, but codes as 17 and 74 can be used to identify cohorts to study outcome of AAV after transplantation(7)

Epidemiological studies in AAV have found incidence rates between 15 and 23 cases per million per year in recent publications(15-17). Data from the US suggest the rates being substantially lower among Afro-Americans(18). Apart from that there are no convincing data that the incidence of AAV varies among different countries except what can be explained by age differences. However, antigen specificity and disease phenotype do vary between populations(19). Figures from England, Japan and Sweden indicate the incidence of ANCA associated nephritis (AAN) to be 15 per million(16, 19). The long term risk of end-stage renal disease (ESRD) among AAN patients seems to be around $30 \%$ (or $20 \%$ among all AAV patients) $(2,9,20)$, figures which infer an incidence of ESRD caused by AAV of 5 per million. The mean age of patients with AAN in both Sweden and Japan is close to 70 years and there is lag time from diagnosis to dialysis dependency. As transplantation is less common above the age of 70 and rare over 75 a substantial portion of AAV patients with ESRD will not be eligible for renal transplantation. In a study by Allen et al only $37 \%(22 / 59)$ of AAV patients with ESRD actually received a transplant even though the median age in that study was relatively low (about 52 a start of renal replacement)(9). A reasonable estimate would be that incidence renal transplantation in the industrialized world is in the range of 1-2 per million; and that 1-3\% of all transplant recipients have $A A V$, in countries like Sweden were the rate of transplantation is around 50 per million. 


\section{Graft function and patient survival after transplantation}

There are several publications reporting on graft and patient survival in $\operatorname{AAV}(3,5-12)$. A general theme in these reports is that AAV patients exhibit survival rates similar to other non-diabetic transplant patients(7). Patient survival rates are as high as $86-93 \%$ five years after transplantation, tending to be better in more recent reports(6). Another consistent finding is that disease phenotype and serotype does not seem to influence survival after transplantation. In a similar manner graft outcomes seem to match the results from transplantation in other renal diseases, without obvious differences between granulomatosis with polyangiitis (GPA) and microscopic polyangiitis (MPA). In a large registry study 10 -year graft survival for GPA patients was $80 \%(7)$, and more recently graft survival censored for death was reported to be as high as $97.9 \%$ after five years in a large multicenter study(6).

\section{Timing of transplantation}

An exacerbation of vasculitis, the induction therapy given to curb it, the transplantation operation as well as the anti-rejection therapy all inflict stress on the immune system increasing the risk for opportunistic infections. It seems logical to postpone transplantation until the patient is in remission and the immune system has recovered from the induction therapy. This also seems to be the common practice in Europe, according to a survey presented by Little et al(10). In the study evidence was also found that patients transplanted $<12$ months after diagnosis of AAV had increased mortality and tended to exhibit vasculopathy on transplant biopsies. Other studies have failed to see any relationship between time since last flare or after onset of dialysis therapy and adverse outcome. Actually even some patients transplanted with ongoing disease activity have been reported to do well(11).

\section{Relapse after transplantation}

$A A V$ is a relapsing disease. About $50 \%$ of patients experience a relapse within 5 years of diagnosis, and the risk is a major concern with respect to transplantation(21). A renal relapse can rapidly 
destroy a graft and confront the patient with substantial risks for additional morbidity(22). These hazards, however, should not be exaggerated and must be balanced with the situation if no transplantation is performed. It is important to keep in mind that extra-renal relapses occur also during dialysis and that most renal relapses respond to therapy. Schmitt et al. compared the risk for relapse before and after transplantation in a cohort of 20 GPA patients and found the overall relapse rate to be higher during dialysis ( 0.3 per year versus 0.1 per year) as compared to after transplantation(11). A more recent study, focusing on MPA patients, also found more relapses during dialysis than after transplantation(23). Several attempts have been made to quantify the risk of relapse of AAV after transplantation. In a pooled analysis published in 1999 we found that $17 \%$ of the patients experienced a relapse, the mean follow-up in these cases was 31 months, which roughly can be transformed to a rate of 0.07 per year(4). Two recent large multicenter reports found substantially lower relapse rate, 0.02 and 0.01 respectively $(6,10)$. Geetha et al suggest this to be due to a better effect of modern anti-rejection therapy to prevent relapses(6). Another possibility would be that patients today tend to receive better induction therapy for their AAV before transplantation. In earlier published cohorts some relapsing patients had not received any induction therapy(12) and as mentioned above there are also reports of patients having received a transplant during ongoing granulomatous inflammation(11).

\section{The role of ANCA testing}

Despite its limitations ANCA is a commonly used biomarker for disease activity in AAV(24). It has been shown that persistent ANCA-positivity during remission is a risk factor for relapse(25). Consequently it would not be surprising if ANCA-positivity at the time of transplantation would be a risk factor for later relapses. This is, however, not a uniform finding in published reports. In a pooled analysis we did not find any statistically significant relationship between ANCA-positivity at transplantation and subsequent relapses(4). Elmedhem et al reported on eight patients, 4 being positive and 4 negative at transplantation, two relapses were recorded both in patients being ANCA- 
negative at transplantation(3). On the other hand Geetha et al. found ANCA at time of transplantation to be a risk factor for relapse(6). In many patients with flares, a rise in ANCA is a late event, so if ANCA is less helpful for prediction it can still help to distinguish relapses from other causes of renal impairment. Patients who are ANCA-positive at time of diagnosis will in most cases also be positive at time of relapse and ANCA levels will be increased compared to remission, at least when analyzed with sensitive assays such as capture PR3-ANCA ELISA(26). The ability of ANCA-tests to confirm a flare does not seem to be blunted after transplantation, positive tests at time of relapse was noted in $3 / 3(8), 5 / 5(23), 2 / 2(9)$ and $7 / 7(6)$ cases. Such a use of ANCA testing naturally requires ANCA to be tested on a regular basis during remission, in order to facilitate interpretation of test results at time of a suspected flare.

\section{Treatment of relapses}

The total number of AAV exacerbations after transplantation described in the literature is still low, and no prospective trials have been reported. Treatments recorded in case series include most therapies used for AAV patients who have not been transplanted, such as corticosteroids alone, increased basal immunosuppression, cyclophosphamide, rituximab and various combinations of these agents $(3,4,6,9,12,22,23,27)$. Recurrent disease in the grafts seems to respond well to cyclophosphamide in most reported cases. However, in the RAVE trial(28), rituximab exhibited superior ability to induce complete remission in relapsing patients as compared to cyclophosphamide. As disease activity after transplantation always is relapse, rituximab is from a theoretical standpoint an attractive alternative. Furthermore renal transplant recipients already have a substantially increased risk of malignancies making avoidance of cyclophosphamide prudent(29).

\section{Concluding remarks}

Renal transplantation is and should be the treatment of choice for end-stage renal disease also for patients with AAV. Transplanted AAV patients exhibit a survival equal or superior to patients with 
other renal diseases, and very few grafts are lost due to recurrence of vasculitis. There is, however, a constant threat of relapse and today there are no reliable clinical or biochemical predictors, but the rate of relapse seems to be lower after transplantation as compared to dialysis treatment. Changes in ANCA levels can most probably help to distinguish relapses from other causes of graft dysfunction. Relapses should be treated with increases in immunosuppressive therapy, but the best way to tailor therapy remains unknown.

\section{References}

1.

Wolfe RA, Ashby VB, Milford EL, Ojo AO, Ettenger RE, Agodoa LY, et al. Comparison of mortality in all patients on dialysis, patients on dialysis awaiting transplantation, and recipients of a first cadaveric transplant. The New England journal of medicine. [Comparative Study

Research Support, U.S. Gov't, P.H.S.]. 1999 Dec 2;341(23):1725-30.

2. Nachman PH, Hogan SL, Jennette JC, Falk RJ. Treatment response and relapse in antineutrophil cytoplasmic autoantibody-associated microscopic polyangiitis and glomerulonephritis. J Am Soc Nephrol. 1996 Jan;7(1):33-9.

3. Elmedhem A, Adu D, Savage CO. Relapse rate and outcome of ANCA-associated small vessel vasculitis after transplantation. Nephrology, dialysis, transplantation : official publication of the European Dialysis and Transplant Association - European Renal Association. [Research Support, Non-U.S. Gov't]. 2003 May;18(5):1001-4.

4. Nachman PH, Segelmark M, Westman K, Hogan SL, Satterly KK, Jennette JC, et al. Recurrent ANCA-associated small vessel vasculitis after transplantation: A pooled analysis. Kidney Int. 1999 Oct;56(4):1544-50.

5. Nyberg G, Akesson P, Norden G, Wieslander J. Systemic vasculitis in a kidney transplant population. Transplantation. 1997 May 15;63(9):1273-7.

6. Geetha D, Eirin A, True K, Valentina Irazabal M, Specks U, Seo P, et al. Renal transplantation in antineutrophil cytoplasmic antibody-associated vasculitis: a multicenter experience. Transplantation. [Multicenter Study]. 2011 Jun 27;91(12):1370-5.

7. Schmitt WH, van der Woude FJ. Organ transplantation in the vasculitides. Current opinion in rheumatology. [Review]. 2003 Jan;15(1):22-8.

8. Haubitz M, Kliem V, Koch KM, Nashan B, Schlitt HJ, Pichlmayr R, et al. Renal transplantation for patients with autoimmune diseases: single-center experience with 42 patients. Transplantation. 1997 May 15;63(9):1251-7.

9. Allen A, Pusey C, Gaskin G. Outcome of renal replacement therapy in antineutrophil cytoplasmic antibody-associated systemic vasculitis. Journal of the American Society of Nephrology : JASN. [Comparative Study

Research Support, Non-U.S. Gov't]. 1998 Jul;9(7):1258-63.

10. Little MA, Hassan B, Jacques S, Game D, Salisbury E, Courtney AE, et al. Renal transplantation in systemic vasculitis: when is it safe? Nephrology, dialysis, transplantation : official publication of the European Dialysis and Transplant Association - European Renal Association. [Research Support, Non-U.S. Gov't]. 2009 Oct;24(10):3219-25.

11. Schmitt WH, Haubitz M, Mistry N, Brunkhorst R, Erbsloh-Moller B, Gross WL. Renal transplantation in Wegener's granulomatosis. Lancet. 1993 Oct 2;342(8875):860. 

patients with antineutrophil cytoplasmic autoantibody-associated vasculitis following cadaveric kidney transplantation. American journal of kidney diseases : the official journal of the National Kidney Foundation. [Case Reports

Comparative Study]. 1997 Jan;29(1):96-102.

13. Basu N, Watts R, Bajema I, Baslund B, Bley T, Boers M, et al. EULAR points to consider in the development of classification and diagnostic criteria in systemic vasculitis. Annals of the rheumatic diseases. [Consensus Development Conference

Research Support, Non-U.S. Gov't]. 2010 Oct;69(10):1744-50.

14. Weidemann S, Andrassy K, Ritz E. ANCA in haemodialysis patients. Nephrology, dialysis, transplantation : official publication of the European Dialysis and Transplant Association European Renal Association. 1993;8(9):839-45.

15. Watts RA, Lane SE, Scott DG, Koldingsnes W, Nossent H, Gonzalez-Gay MA, et al. Epidemiology of vasculitis in Europe. Ann Rheum Dis. 2001 Dec;60(12):1156-7.

16. Mohammad AJ, Jacobsson LT, Westman KW, Sturfelt G, Segelmark M. Incidence and survival rates in Wegener's granulomatosis, microscopic polyangiitis, Churg-Strauss syndrome and polyarteritis nodosa. Rheumatology (Oxford). 2009 Dec;48(12):1560-5.

17. Ormerod AS, Cook MC. Epidemiology of primary systemic vasculitis in the Australian Capital Territory and south-eastern New South Wales. Intern Med J. [Comparative Study]. 2008 Nov;38(11):816-23.

18. Cao Y, Schmitz JL, Yang J, Hogan SL, Bunch D, Hu Y, et al. DRB1*15 allele is a risk factor for PR3-ANCA disease in African Americans. Journal of the American Society of Nephrology : JASN. [Research Support, N.I.H., Extramural]. 2011 Jun;22(6):1161-7.

19. Watts RA, Scott DG, Jayne DR, Ito-Ihara T, Muso E, Fujimoto S, et al. Renal vasculitis in Japan and the UK--are there differences in epidemiology and clinical phenotype? Nephrol Dial Transplant. 2008 Dec;23(12):3928-31.

20. Booth AD, Almond MK, Burns A, Ellis P, Gaskin G, Neild GH, et al. Outcome of ANCAassociated renal vasculitis: a 5-year retrospective study. Am J Kidney Dis. 2003 Apr;41(4):776-84.

21. Walsh M, Flossmann O, Berden A, Westman K, Hoglund P, Stegeman C, et al. Risk factors for relapse of antineutrophil cytoplasmic antibody-associated vasculitis. Arthritis and rheumatism. [Research Support, Non-U.S. Gov't]. 2012 Feb;64(2):542-8.

22. Steinman TI, Jaffe BF, Monaco AP, Wolff SM, Fauci AS. Recurrence of Wegener's granulomatosis after kidney transplantation. Successful re-induction of remission with cyclophosphamide. The American journal of medicine. [Case Reports]. 1980 Mar;68(3):458-60. 23. Merino JL, Galeano C, Espejo B, Rivera M, Fernandez-Lucas M, Caldes S, et al. A retrospective study on outcome of microscopic polyangiitis in chronic renal replacement therapy. Nephrology, dialysis, transplantation : official publication of the European Dialysis and Transplant Association - European Renal Association. 2011 Apr;26(4):1360-6.

24. Nowack R, Grab I, Flores-Suarez LF, Schnulle P, Yard B, van der Woude FJ. ANCA titres, even of IgG subclasses, and soluble CD14 fail to predict relapses in patients with ANCA-associated vasculitis. Nephrology, dialysis, transplantation : official publication of the European Dialysis and Transplant Association - European Renal Association. 2001 Aug;16(8):1631-7.

25. Sanders JS, Huitma MG, Kallenberg CG, Stegeman CA. Prediction of relapses in PR3ANCA-associated vasculitis by assessing responses of ANCA titres to treatment. Rheumatology (Oxford). 2006 Jun;45(6):724-9.

26. Segelmark M, Phillips BD, Hogan SL, Falk RJ, Jennette JC. Monitoring proteinase 3 antineutrophil cytoplasmic antibodies for detection of relapses in small vessel vasculitis. Clin Diagn Lab Immunol. 2003 Sep;10(5):769-74. 
27.

Geetha D, Seo P, Specks U, Fervenza FC. Successful induction of remission with rituximab for relapse of ANCA-associated vasculitis post-kidney transplant: report of two cases. Am J Transplant. [Case Reports

Research Support, Non-U.S. Gov't]. 2007 Dec;7(12):2821-5.

28. Stone JH, Merkel PA, Spiera R, Seo P, Langford CA, Hoffman GS, et al. Rituximab versus cyclophosphamide for ANCA-associated vasculitis. N Engl J Med. 2010 Jul 15;363(3):221-32.

29. Rama I, Grinyo JM. Malignancy after renal transplantation: the role of immunosuppression. Nature reviews Nephrology. [Review]. 2010 Sep;6(9):511-9. 